\title{
Eficiência do tratamento combinado de impregnação e termorretificação nas propriedades da madeira de pinus
}

\author{
Enhancing pine wood properties by two- \\ step impregnation-heat treatment
}

\begin{abstract}
Aline Krolow Soares ${ }^{1}$, Pedro Henrique Gonzalez de Cademartori ${ }^{2}$, Dmitry Victorovitch Evtyugin ${ }^{3}$, Paula Zanatta ${ }^{1}$, Darci Alberto Gatto ${ }^{1}$
\end{abstract}

\footnotetext{
${ }^{1}$ UFPel - Universidade Federal de Pelotas, Centro de Desenvolvimento Tecnológico (CDTec), Rua Gomes Carneiro, $\mathrm{n}^{\circ} 1$, CEP: 96010-610, Campus Anglo, Pelotas, Rio Grande do Sul, Brasil.

${ }^{2}$ UFPR - Universidade Federal do Paraná, Departamento de Engenharia e Tecnologia Florestal (DETF), Av. Pref. Lothário Meissner, nº32, CEP: 80210-170, Campus Jardim Botânic, Curitiba, Paraná, Brasil.

${ }^{3}$ UA - Universidade de Aveiro, Departamento de Química (DQA), CEP: 3810-193, Campus Universitário de Santiago, Aveiro, Portugal.

e-mail: alinekrolowsoares@yahoo.com.br, pedrocademartori@gmail.com,dmitrye@ua.pt, zanatta_paula@hotmail.com, darcigatto@yahoo.com
}

\section{RESUMO}

O estudo teve como objetivo avaliar a eficiência do tratamento combinado de impregnação com resina seguido de termorretificação em madeiras de Pinus sp. Para tal, foram selecionadas ao acaso quinze peças cúbicas de $15 \mathrm{~mm}$ de aresta. A primeira etapa consistiu na impregnação de resina termoplástica a base de breu e a segunda etapa na termorretificação das peças de madeira a $180^{\circ} \mathrm{C}$ durante 30 minutos. As impregnações foram realizadas por dois métodos distintos: vácuo-imersão (VT) e pressão hiperbárica (HBT). O período de vácuo foi 15 min e tempo total da impregnação de três horas. A pressão empregada foi de 6000bar no HBT. Os tratamentos foram analisados por meio da retenção de solução e perda de massa. A avaliação das propriedades físicas e térmicas deu-se por meio de análise da taxa de absorção de água, inchamentos radial, tangencial e volumétrico, molhabilidade e análise termogravimétrica. A resistência biológica foi determinada a partir de ensaios de apodrecimento acelerado. Os resultados obtidos denotam que os tratamentos se mostraram eficientes, com destaque para o tratamento HBT. O ângulo de contato mostrou-se constante ao longo do tempo após os tratamentos, ao passo que as amostras controle apresentaram comportamento hidrofílico. A resistência biológica das madeiras tratadas por VT e HBT aumentou substancialmente em relação a madeira in natura de Pinus sp. Conclui-se que o tratamento combinado apresenta potencial como alternativa para incremento das propriedades físicas da madeira de Pinus sp.

Palavras-chave: tratamento térmico, resina, higroscopicidade, hidrofobicidade.

\section{ABSTRACT}

This study aimed to evaluate the efficiency of the combined treatment of rosin impregnation followed by heat treatment in Pinus sp wood. Fifteen cubic samples of $15 \mathrm{~mm}$ edge were randomly selected. The first step consisted of the impregnation of thermoplastic resin based on rosin and the second step was the heat treatment of wood at $180^{\circ} \mathrm{C}$ for 30 minutes. The impregnations were performed by two different methods: vacuumimmersion (VT) and hyperbaric pressure (HBT). The vacuum period was $15 \mathrm{~min}$ and total impregnation time was three hours. The pressure in the HBT treatment was $6000 \mathrm{bar}$ for $15 \mathrm{~min}$. The treatments were analyzed through the solution retention and mass loss. The evaluation of the physical characteristics was done through the analysis of water absorption rates, radial, tangential, volumetric swellings, wettability and thermogravimetric analysis. The decay resistance was evaluated by accelerated decay tests. The results showed the efficiencyof the treatments, especially the HBT treatment. The contact angle was constant over the timefor the treated wood samples, whereas, the untreated wood sampled presented hydrophilic behavior. The decay resistance of treated wood with VT and HBT treatments increased in relation to the untreated Pinus sp. wood. 
The combined treatment is a potential alternative to improve the physical properties of Pinus sp. wood.

Keywords: heat treatment, resin, hygroscopicity, hydrophobicity.

\section{INTRODUÇÃO}

A ampla utilização da madeira em setores industriais, tais como polpa e papel, energia da biomassa e painéis reconstituídos coexiste com as limitações deste material, especialmente o elevado nível de higroscopicidade e anisotropia, heterogeneidade e suscetibilidade à biodegradação em comparação a outros materiais. Nesse contexto, a modificação das propriedades da madeira por meio de tratamentos de superfície ou impregnação é necessária a fim de contornar tais limitações da madeira quando em serviço.

Baseado nestes aspectos, tratamentos alternativos aos mais tradicionais têm sido investigados, de maneira a proporcionar variações positivas nas propriedades da madeira, que vão desde o aumento da resistência biológica até o incremento da estabilidade dimensional. Entre os estudos, ressalta-se a polimerização in situ com monômeros [1] [2], termorretificação [3] [4] e impregnação com produtos naturais [5] [6] [7].

A termorretificação é um processo de exposição de peças de madeira a temperaturas entre $120^{\circ} \mathrm{C}$ e $280^{\circ} \mathrm{C}$, a qual viabiliza a modificação das propriedades físicas, mecânicas e biológicas, devido a alterações em sua constituição química. Contudo, a eficiência deste processo no aumento da estabilidade dimensional [8] [9] e na redução da higroscopicidade [10] [11] ocorre concomitantemente à perda de massa [12] e à redução da resistência mecânica [12] [13] [14], em razão dos polímeros que constituem a parede celular da madeira[4] [15] [16].

A impregnação com produtos naturais abrange o uso de extratos provenientes da própria madeira,bem como o uso de resíduos com baixa ou nenhuma reutilização por parte das indústrias, como os bio-óleos, por exemplo. Nesse caso, busca-se a utilização de materiais oriundos de resíduos não aproveitados que favoreçam e incrementem as propriedades da madeira, o que reflete preocupação com a sustentabilidade.

Alguns exemplos são a impregnação de borato seguido de termorretificação [17] e melamina-ureia seguido de termorretificação [18] [19]. Esses tratamentos são geralmente conduzidos inicialmente em autoclave e posteriormente em estufa com circulação de ar, proporcionando à madeira uma exposição completa às condições úmidas e secas, com o objetivo de ampliar suas propriedades físico-químicas e mecânicas.

Dentre os materiais promissores encontra-se o breu, produto do processo de resinagem de árvores de Pinus sp. O breu, devido à sua composição de ácidos resínicos e abiéticos [20] [21], confere resistência a organismos xilófagos e aumenta o caráter hidrofóbico da madeira [22]. Portanto, é um material com grande potencial para ser empregado na indústria de tratamento de madeira.

A associação dos tratamentos de impregnação e termorretificação, combinados com a utilização de um produto natural como o breu representa uma promissora área a ser investigada, de forma a ser utilizada a nível industrial. $\mathrm{O}$ presente estudo visa avaliar o efeito do tratamento combinado de impregnação com vácuo (VT) e com pressão hiperbárica (HBT) de resina termoplástica a base de breu seguida de termorretifiação, nas propriedades físicas, químicas e biológicas da madeira de Pinus sp.

\section{MATERIAIS E MÉTODOS}

\subsection{Seleção e preparo do material}

A madeira utilizada no presente estudo proveio de uma população de Pinus sp. localizado na cidade de Santa

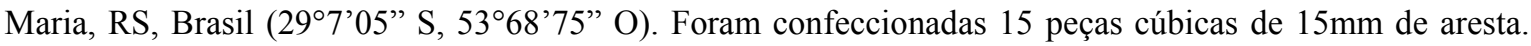
Após a confecção, os corpos de prova foram acondicionados em câmara climática $\left(23^{\circ} \mathrm{C}\right.$ e $50 \%$ UR) até a obtenção do teor de umidade de equilíbrio. Cada um dos tratamentos empregados e as amostras controle caracterizaram-se por apresentar cinco corpos de prova.

\subsection{Tratamentos combinados}

O método empregado foi de um tratamento combinado com duas etapas. A primeira etapa consistiu na impregnação de resina termoplástica à base de breu, e a segunda etapa na termorretificação das peças de madeira a $180^{\circ} \mathrm{C}$. Dessa forma, o experimento foi delineado com as amostras controle (C), o tratamento de impregnação em sistema de vácuo seguido por termorretificação (VT) e o tratamento que consistiu na impregnação hiperbárica e termorretificação (HBT). Esses tratamentos foram efetuados a fim de que pudesse ser feita uma comparação entre um método simples de tratamento empregado nas indústrias, adaptado para reali- 
zação em laboratório (VT) e um tratamento hiperbárico, que alcança pressões elevadas e assim, a impregnação pode ser efetuada em menor tempo e com maior eficiência (HBT).

\section{Primeira etapa: Impregnação com resina termoplástica}

O tratamento de impregnação por vácuo (VT) consistiu na aplicação de vácuo inicial de 15 min, seguido pela adição de resina termoplástica a base de tolueno. Estas, foram solubilizadas a uma concentração de $8,2 \% \mathrm{w} / \mathrm{v}$, próximo ao limite de saturação da resina. Após este processo, as amostras de madeira foram transferidas para frascos que foram preenchidos com a solução. As amostras ficaram imersas durante três horas na solução e, por fim, foram secas em estufa laboratorial com ventilação a $40^{\circ} \mathrm{C}$ por 12 horas.

O tratamento de impregnação por pressão hiperbárica (HBT) foi realizado por meio da aplicação de vácuo inicial (15min) seguido pela adição da resina termoplástica. Posteriormente as amostras e a solução foram transferidas para frascos e levadas a autoclave industrial de pressão hiperbárica. Foi aplicada pressão de 6000bar a temperatura ambiente $\left(22^{\circ} \mathrm{C}\right)$ por $15 \mathrm{~min}$. $\mathrm{O}$ tempo transcorrido desde o momento em que as amostras foram postas nos frascos até a retirada foi de três horas. Após este período, as amostras submetidas aos tratamentos HBT foram secas em estufa laboratorial com ventilação a $40^{\circ} \mathrm{C}$ por 12 horas.

A retenção de solução (Rs) foi calculada para avaliar efetivamente a porção de solução (resina termoplástica a base de breu) que permaneceu retida na madeira, de acordo com procedimento descrito na norma UNE EN 351-1 [23].

\section{Segunda etapa: Termorretificação}

As amostras impregnadas foram submetidas a tratamentos de termorretificação a $180^{\circ} \mathrm{C}$ em uma estufa laboratorial com exaustão de ar exterior, pré-aquecida por 30min. Após a realização dos tratamentos, todo o material foi armazenado em local com temperatura e umidade controladas $\left(23^{\circ} \mathrm{C}\right.$ e $\left.50 \% \mathrm{UR}\right)$, para estabilização do teor de umidade.A perda de massa (PM) após a termorretificação foi determinada a partir da variação de massa após a primeira etapa, de impregnação, em relação à massa final, após a segunda etapa do tratamento.

\subsection{Absorção de água e estabilidade dimensional}

A estabilidade dimensional da madeira de Pinus sp. foi mensurada por meio das variáveis taxa de absorção de água $\left(\mathrm{T}_{\mathrm{H} 2 \mathrm{O}}\right)$, inchamento volumétrico $\left(\alpha_{\mathrm{v}}\right)$, inchamento linear $\left(\alpha_{\mathrm{r}}\right.$ e $\left.\alpha_{\mathrm{t}}\right)$ e anisotropia (A). Para tanto, os corpos de prova das amostras controle e tratadas foram totalmente imersos em água destilada por um período de 24 horas. Dados de dimensão (radial, tangencial e axial) e massa do material foram coletados antes e após a imersão. Posteriormente, os corpos de prova foram secos em estufa laboratorial a uma temperatura de $103 \pm 2{ }^{\circ} \mathrm{C}$ para mensuração dos mesmos parâmetros em condições anidras. Com os dados coletados, foi possível avaliar os parâmetros de estabilidade dimensional das peças tratadas e não tratadas, conforme as equações $1,2,3,4$ e 5 .

$$
\begin{aligned}
& T_{H 2 O}=\frac{m_{u}-m_{s}}{m_{s}} \times 100 \\
& \alpha_{V}=\frac{v_{u}-v_{s}}{v_{s}} \times 100 \\
& \alpha_{R}=\frac{R_{u}-R_{s}}{R_{s}} \times 100 \\
& \alpha_{T}=\frac{T_{u}-T_{s}}{T_{s}} \times 100 \\
& A=\frac{\alpha_{R}}{\alpha_{T}}
\end{aligned}
$$

Em que: $\mathrm{m}_{\mathrm{u}}=$ massa dos corpos de prova saturados $(\mathrm{g}) ; \mathrm{m}_{\mathrm{s}}=$ massa dos corpos de prova secos em estufa a teor de umidade de $0 \%(\mathrm{~g}) ; \mathrm{v}_{\mathrm{u}}=$ volume dos corpos de prova saturados $\left(\mathrm{cm}^{3}\right) ; \mathrm{v}_{\mathrm{s}}=$ volume dos corpos de prova secos em estufa $\left(\mathrm{cm}^{3}\right)$; Tu= dimensão na direção tangencial saturada $(\mathrm{cm})$; Ts= dimensão na direção tangencial anidra $(\mathrm{cm}) ; \mathrm{Ru}=$ dimensão na direção radial saturada $(\mathrm{cm})$; Rs= dimensão na direção radial anidra $(\mathrm{cm})$.

\subsection{Molhabilidade}

A molhabilidade das madeiras tratadas e não tratadas foi avaliada através da técnica de ângulo de contato. Para essa análise utilizou-se um goniômetro Biolin Scientific Inc., modelo Theta Light TL 101. Os ensaios 
foram realizados por meio do método de gota séssil, a partir da disposição de 5 gotículas com $5 \mu$ l de água deionizada em cinco locais distintos sob o plano tangencial da madeira. O comportamento cinético da gotícula de água na superfície da madeira foi realizado nos instantes 5, 25, 45, 65 e 85 segundos após o contato da gotícula com a superfície da madeira.

\subsection{Análise termogravimétrica}

A análise termogravimétrica (TGA) foi utilizada para avaliar a interação (física ou química) entre a resina termoplástica e a madeira e também para avaliar a estabilidade térmica. A curva termogravimétrica (TGA) e a derivada (DTG) foram obtidas utilizando-se um equipamento DTG-60 da Shimadzu. Para isso, empregouse atmosfera inerte de nitrogênio com fluxo de gás de $20 \mathrm{ml} . \mathrm{min}^{-1}$, intervalo de temperatura entre 25 e $600^{\circ} \mathrm{C}$ e taxa de aquecimento de $10^{\circ} \mathrm{C} \cdot \mathrm{min}^{-1}$. A massa das amostras variou entre 5-8mg e um recipiente de platina foi utilizado para alocar as amostras no equipamento.

\subsection{Ensaio de apodrecimento acelerado}

O ensaio de apodrecimento acelerado foi conduzido conforme a norma ASTM D2017 [24]. Foi utilizada uma colônia do fungo de podridão-branca Ganoderma applanatum crescida em meio extrato de malte ágar por cerca de 21 dias, sob incubação com temperatura a $25 \pm 3^{\circ} \mathrm{C}$ e fotoperíodo de 12 horas. Foram ensaiados quatro amostras por tratamento mais as controle, as quais permaneceram em incubação por um período de 150 dias [25]. Após este período, foi calculada a perda de massa das amostras.

\subsection{Análise estatística dos dados}

Para a avaliação dos resultados foi empregado o método de delineamento experimental por meio de análise de variância fatorial (ANOVA), pela qual foi analisada a interação entre as variáveis de estabilidade dimensional e o fator tratamento. A comparação das médias foi realizada a por meio do teste $\mathrm{F}$, a $5 \%$ de probabilidade de erro. Para os fatores em que ocorreram interações significativas e em caso de rejeição da hipótese nula, foram realizados testes de média HSD Tukey e avaliados a 5\% de probabilidade de erro.

\section{RESULTADOS E DISCUSSÃO}

\subsection{Parâmetros do tratamento}

A retenção de solução do tratamento $\operatorname{HBT}(8,11 \%)$ foi bastante superior à retenção do tratamento VT $(6,45 \%)$. Isso evidencia e justifica a utilização da pressão hiperbáricaque promove a entrada da solução nos microporos da madeira, permitindo maior eficiência [26] [27]. Lesar et al. [28] afirmam que alguns dos fatores que influenciam na retenção da solução são a densidade e a viscosidade do produto impregnado, no caso das ceras ocorre formação de barreiras nas cavidades celulares que impedem a entrada de solventes (água) para o interior das células, reduzindo assim a fixação uniforme do produto.

A perda de massa obtida foi de 9,32 e 9,95\% para os tratamentos HBT e VT, respectivamente. Está relacionada a degradação térmica da madeira e da resina termoplástica aplicada, suscetível a degradação devido à altas temperaturas. Conforme Caetano [29], o breu começa a amolecer, sem se degradar, na temperatura de $77^{\circ} \mathrm{C}$. Durante a termorretificação da madeira a $180^{\circ} \mathrm{C}$, ocorreu a degradação de certos componentes majoritários da parede celular [30] [31], principalmente as hemiceluloses. A modificação na estrutura química em conjunto com a modificação de estado físico da resina termoplástica a base de breu promove um maior acúmulo do material nas cavidades porosas da madeira. Este material pode preencher os espaços vazios [28] ou então associar-se com a madeira, como observado por Mattos et al. [2], em que ocorreu polimerização in situ entre a madeira e o metacrilato após cura a $60^{\circ} \mathrm{C}$.

\subsection{Absorção de água e estabilidade dimensional}

A absorção de água $\left(\mathrm{T}_{\mathrm{H} 2 \mathrm{O}}\right)$ para a madeira tratada foi significativamente menor do que nas amostras de controle. A impregnação da resina seguida de tratamento térmico reduziu cerca de 3 vezes a capacidade da madeira para reter água. No entanto, o uso de pressão hiperbárica não afetou o $\mathrm{T}_{\mathrm{H} 2 \mathrm{O}}$ quando comparado ao método de imersão simples. A menor absorção de água provavelmente ocorre devido à degradação das hemiceluloses após tratamento térmico a $180^{\circ} \mathrm{C}$ e a um efeito sinérgico da impregnação de resina e tratamento térmico, como anteriormente assumido por Wang et al. [32] para impregnação de cera seguido de tratamento térmico de madeira de pinho do sul (Tabela 1). 
Tabela 1: ANOVA entre os parâmetros de estabilidade dimensional e o fator tratamento.

\begin{tabular}{cccccc}
\hline TRATAMENTO & $\mathbf{T}_{\mathbf{H} 20}$ & $\boldsymbol{\alpha}_{\mathrm{r}}$ & $\boldsymbol{\alpha}_{\mathrm{t}}$ & $\boldsymbol{\alpha}_{\mathbf{v}}$ & $\mathbf{A}$ \\
\hline \multirow{2}{*}{$\mathrm{C}$} & $91,16 \mathrm{~b}$ & $4,58 \mathrm{a}$ & $8,02 \mathrm{a}$ & $14,76 \mathrm{a}$ & $1,91 \mathrm{a}$ \\
& $(14,41)$ & $(9,75)$ & $(4,63)$ & $(8,84)$ & $(23,40)$ \\
\hline \multirow{2}{*}{$\mathrm{VT}$} & $33,42 \mathrm{a}$ & $4,71 \mathrm{a}$ & $8,12 \mathrm{ab}$ & $13,51 \mathrm{a}$ & $1,76 \mathrm{a}$ \\
& $(6,45)$ & $(15,32)$ & $(5,75)$ & $(5,87)$ & $(5,27)$ \\
\hline \multirow{2}{*}{$\mathrm{HBT}$} & $32,62 \mathrm{a}$ & $4,49 \mathrm{a}$ & $9,09 \mathrm{~b}$ & $13,13 \mathrm{a}$ & $1,79 \mathrm{a}$ \\
& $(7,33)$ & $(13,81)$ & $(8,58)$ & $(6,95)$ & $(18,15)$ \\
\hline \multirow{2}{*}{ Teste F } & $72,10^{*}$ & $0,41^{\mathrm{ns}}$ & $4,84^{*}$ & $2,95^{\mathrm{ns}}$ & $0,27^{\mathrm{ns}}$ \\
\hline
\end{tabular}

Em que: $\mathrm{T}_{\mathrm{H} 2 \mathrm{O}}=$ absorção de água; $\alpha \mathrm{r}=$ inchamento radial; $\alpha \mathrm{v}=$ inchamento volumétrico; $\mathrm{A}=$ anisotropia. Valores entre parênteses referem-se ao coeficiente de variação. Letras diferentes na coluna indicam diferenças estatísticas significativas entre os tratamentos.

De acordo com Lesar et al. [28], dentre os fatores que comprometem a eficiência de tratamentos que visam contornar problemas como a higroscopicidade, está a interação ocorrida entre as ligações (-OH) presentes nos componentes majoritários da madeira e as moléculas de água. Acredita-se que a resina termoplástica não formou ligações com os grupos -OH disponíveis na madeira, deixando-os livres para ligarem-se com as moléculas de água, como visto por meio da variável de inchamento volumétrico. Nesse sentido, as amostras controle tiveram uma taxa de absorção maior mas um inchamento proporcional às madeiras tratadas, denotando a eficiência da resina termoplástica para impedir a interação com a água.

\subsection{Molhabilidade}

A impregnação de resina seguida por termorretificação melhorou a hidrofobicidade superficial da madeira tratada (Figura 1). $\mathrm{O}$ ângulo de contato (CA) permaneceu constante em torno de $80^{\circ}$ em função do tempo para os tratamentos VT e HBT. Como era esperado, o CA da madeira sem tratamento diminuiu em torno de $35 \%$ após $85 \mathrm{~s}$, principalmente até os primeiros $45 \mathrm{~s}$.

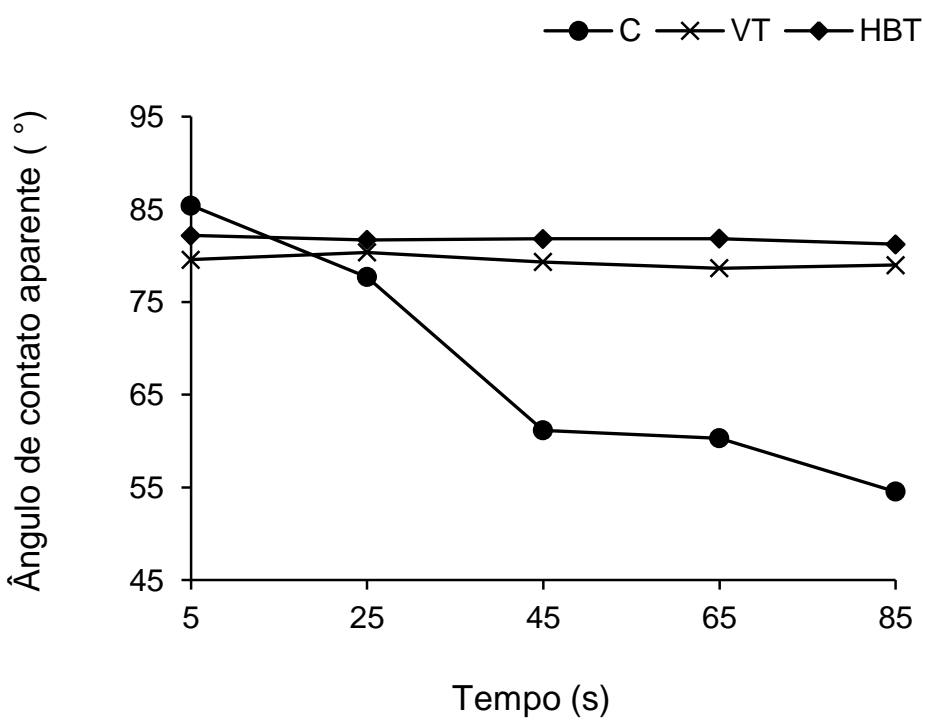

Figura 1: Cinética do ângulo de contato aparente para as madeiras de Pinus sp.

A estabilidade do CA para a madeira tratada está relacionada a inativação da superfície proporcionado pela eliminação de sítios hidroxila da superfície pelo tratamento térmico e bloqueio de microestruturas responsáveis pela absorção de água da madeira de pinus a partir da impregnação com a resina termoplástica.

Essefenômeno de inativação afeta apenas uma fina camada externa da madeira [33] e pode reduzir as forças de atração na superfície da madeira, acarretando em diminuição da sua molhabilidade, tal como obser- 
vado nesse estudo.

De acordo com Yildiz et al. [34], após a madeira ser submetida a tratamentos de termorretificação até cerca de $200^{\circ} \mathrm{C}$, a celulose tende a perder sua porção amorfa, permanecendo intacta a zona cristalina. Nas zonas cristalinas as ligações de hidrogênio são fortes [35] e não permitem rompimento por quaisquer solventes orgânicos. Além disso, assim como verificado na absorção de água, o CA aumentou a repelência do material à água, o qual está associado à formação de uma película de proteção com a resina à base de breu após a termorretificação, assim como um possível aumento da micro/nano rugosidade superficial devido ao tratamento térmico.

\subsection{Análise termogravimétrica}

Os termogramas na Figura 2 ilustram a perda de massa e a derivada (dw/dt) para a resina impregnada e para a madeira de Pinus sp. Até $100^{\circ} \mathrm{C}$, ocorreu a evaporação da água higroscópica presente na madeira. Nota-se que a resina apresentou menores concentrações de água nessa faixa de temperatura pois apresentou menor perda de massa. Na faixa entre 200 e $350^{\circ} \mathrm{C}$ as madeiras controle e tratadas por VT apresentaram comportamento térmico muito semelhante, devido à degradação da hemicelulose, porém o pico endotérmico apresentado na derivada $(\mathrm{dw} / \mathrm{dt})$ das madeiras tratadas por VT ocorreu em uma maior temperatura em relação ao controle, denotando uma maior resistência térmica do polímero em questão. Observa-se a presença de um pico endotérmico entre 400 e $500^{\circ} \mathrm{C}$ na degradação térmica dos tratamentos VT e HBT, diferentemente do observado nas madeiras controle. Isso sugere que as hemiceluloses foram degradadas durante a segunda etapa de termorretificação dos tratamentos combinados.
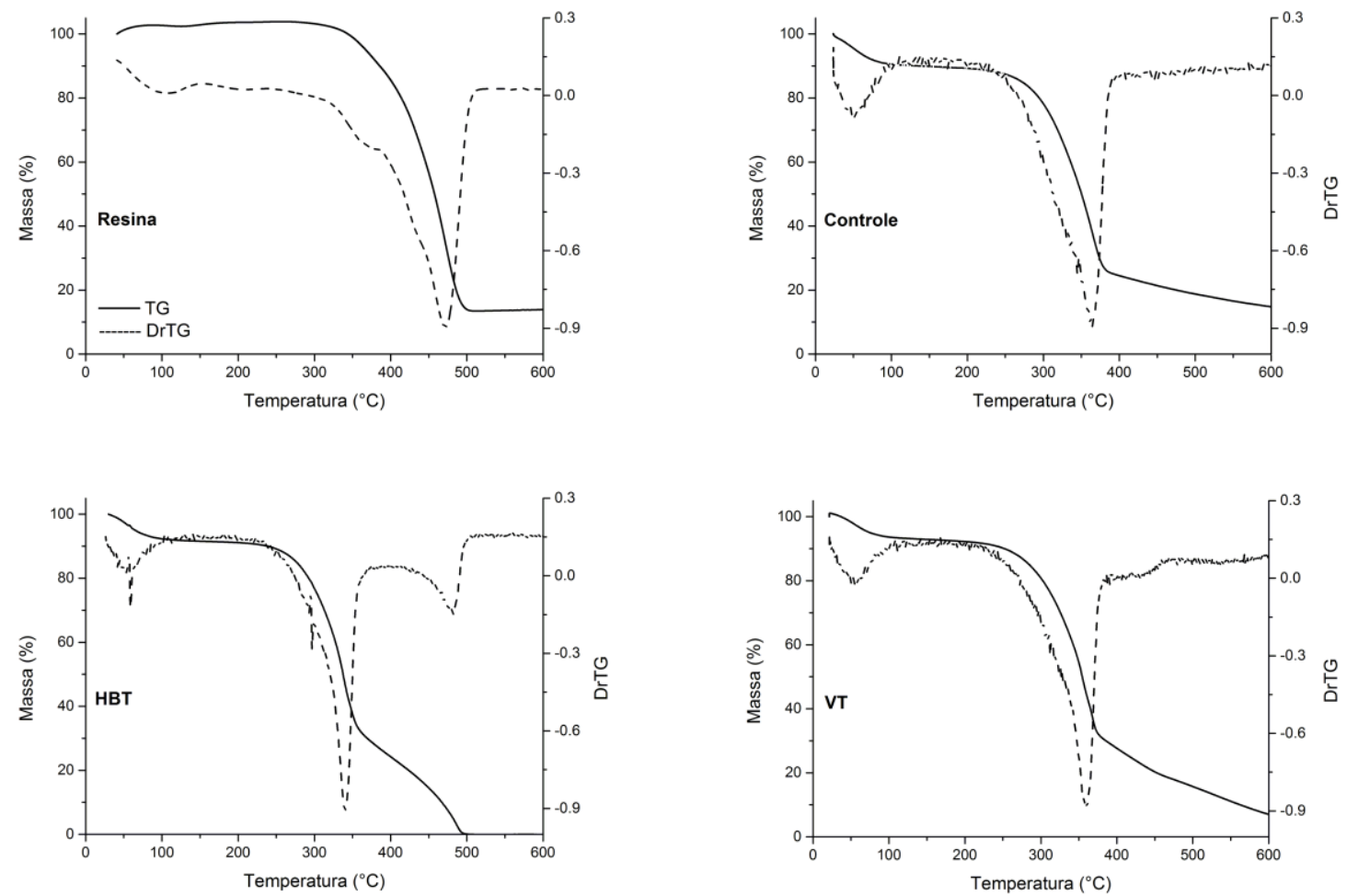

Figura 2: Curvas TGA/DTGA da resina e das madeiras tratadas e não tratada.

As madeiras tratadas por HBT apresentaram degradação térmica distinta da observada na madeira controle e tratadas por VT. Nesse tratamento houve uma nova região de degradação a $500^{\circ} \mathrm{C}$, e a perda de massa chegou quase a $100 \%$ nessa temperatura. Essa degradação a $500^{\circ} \mathrm{C}$ pode estar relacionada com a degradação da resina em madeira impregnada com a mesma, cujas degradações térmicas (intervalo de temperaturas e temperatura de degradação máxima) foram muito parecidas. Já as amostras controle a ficaram com $12 \%$ de massa residual após o ensaio de TGA e as amostras tratadas por VT tiveram $8 \%$ de massa final a $600^{\circ}$ indicando os processos distintos de formação de carvão em amostras. 


\subsection{Ensaio de apodrecimento acelerado}

Após o ensaio de apodrecimento acelerado ficou evidente o sucesso do tratamento combinado. As amostras controle tiveram uma perda de massa de $41,6 \%$ e as amostras HBT e VT tiveram perdas de massa de 9,6 e $8,7 \%$, respectivamente. É possível verificar a severidade dos ataques nas amostras sem nenhum tratamento em relação àquelas dos tratamentos HBT e VT (Figura 3).

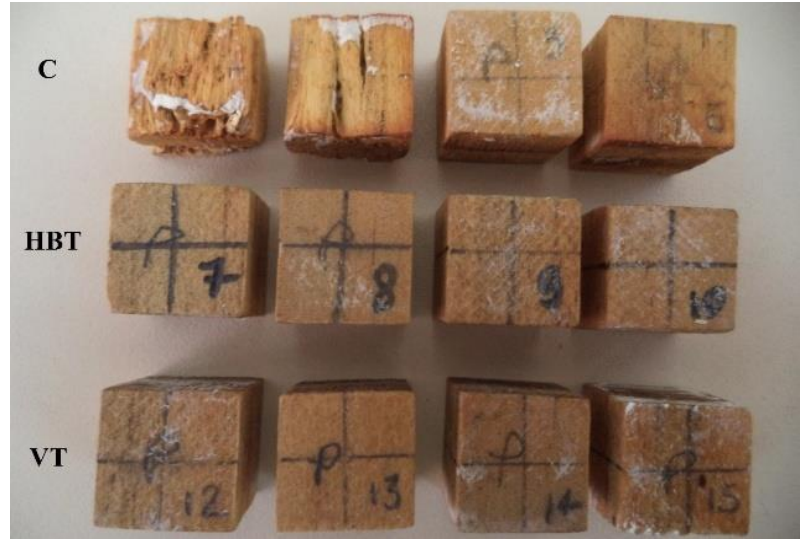

Figura 3: Aspecto das amostras submetidas ao ensaio de apodrecimento acelerado.

Dessa forma, é possível comprovar que a resina termoplástica a base de breu formou uma camada de proteção na superfície da madeira de Pinus sp., impedindo que as enzimas digestivas liberadas pelos fungos de podridão branca ataquem os polímeros constituintes da parede celular. Portanto, as madeiras tratadas com a resina tiveram um aumento na resistência a agentes de podridão branca.

Essa maior resistência biológica em madeiras tratadas pode ser associada com a propriedade física de absorção de água, que também foi inferior em madeiras tratadas. Uma vez que as madeiras tratadas absorvem menos água quando expostas a umidade, os fungos não se desenvolvem com o mesmo vigor pois necessitam de umidade para que ocorra o crescimento dos micélios. Assim, as madeiras tratadas por VT e HBT apresentam um potencial para emprego em nível industrial.

\section{CONCLUSÕES}

Conclui-se que os tratamentos combinados diminuíram a taxa de absorção de água e tornaram a superfície das peças tratadas mais hidrofóbicas. A resistência biológica das madeiras tratadas foi superior àquelas não tratadas, evidenciando o potencial de aplicação do tratamento combinado para melhorar as propriedades físicas e biológicas da madeira de Pinus sp.

\section{AGRADECIMENTOS}

Os autores gostariam de agradecer à Fundação de Amparo à Pesquisa do Rio Grande do Sul (FAPERGS), ao Programa Santander Universidades e à Coordenação de Aperfeiçoamento de Pessoal de Nível Superior (CAPES), processo 00.889.834/0001.08, pelo suporte financeiro.Os autores também gostariam de agradecer o projeto CICECO-Aveiro Institute of Materials, POCI-01-0145-FEDER-007679 (FCT Ref. UID/CTM/50011/2013), financiado por fundos nacionais através da FCT / MEC e quando apropriado Cofinanciado pela FEDER ao abrigo do Acordo de Parceria PT2020.

\section{BIBLIOGRAFIA}

[1] HADI, Y. S., RAHAYU, I. S., DANU, S. "Physical and mechanical properties of ethyl methacrylate impregnated jabon wood", Journal of the Indian Academy of Wood Science, pp. 1-4, 2013/10/25 2013.

[2] MATTOS, B. D., GATTO, D. A., MAGALHÃES, W. L. E. "Compósitos polímero-madeira preparados por polimerização in situ: conceitos, parâmetros de processo e propriedades", Ciência da Madeira, v.6, pp.129-148, 2015.

[3] HILL, C. A. S., RAMSEY, J., KEATING, B., et al., "The water vapour sorption properties of thermally modified and densified wood", Journal of Materials Science, v.47, pp.3191-3197, 2012. 
[4] ESTEVES, B., VIDEIRA, R., PEREIRA, H. "Chemistry and ecotoxicity of heat-treated pine wood extractives", Wood Science and Technology, v.45, pp.661-676, 2011.

[5] HERRERA, R., ERDOCIA, X., PONTE, R. L., et al., "Characterization of hydrothermally treated wood in relation to changes on its chemical composition and physical properties", Journal of Analytical and Applied Pyrolysis, v.107, pp.256-266, 2014.

[6] HUNG, K. C., WU, T. L., CHEN, Y. L., et al., "Assessing the effect of wood acetylation on mechanical properties and extended creep behavior of wood/recycled-polypropylene composites", Construction and Building Materials, v.108, p.139-145, 2016.

[7] SALEM, M. Z. M., ZIDAN, Y. E., MANSOUR, M. M. A., et al., "Antifungal activities of two essential oils used in the treatment of three commercial woods deteriorated by five common mold fungi", International Biodeterioration \& Biodegradation, v.106, pp.88-96, 2016.

[8] KORKUT, S., BEKTAS, I. "The effectsof heart treatment on phisical properties of Uludag fir (Abies bornmuelleriana Mattf.) and Scots pine (Pinus sylvestris L.) wood”, Forest Products Journal, v.58, pp.95-99, 2008.

[9] KOCAEFE, D., SHI, J. L., YANG, D. O., et al., "Mechanical properties, dimensional stability, and mold resistance of heart-treated jack pine and aspen", Forest Products Journal, v.58, pp.88-93, 2008.

[10] BORREGA, M., KÄRENLAMPI, P. P. "Hygroscopicity of heattreated Norway spruce (Picea abies) wood", European Journal of Wood and Wood Products, v.68, p.233-235, 2010.

[11] ESTEVES, B., PEREIRA, H. "Quality assessment of heattreated wood by NIR spectroscopy", Holz als Roh-und Werkstoff, v.66, pp.323-332, 2008.

[12] KOCAEFE, D., PONCSAK, S., TANG, J., et al., "Effect of heat treatment on the mechanical properties of North American jack pine: Thermogravimetric study", Journal of Materials Science, v.45, pp.681-687, 2010.

[13] PFRIEM, A., BUCHELT, B., ZAUER, M., et al., "Comparative analysis of thermally modified and native spruce loaded perpendicular to the grain", European Journal of Wood and Wood Products, v.68, pp.267270, 2010.

[14] ROWELL, R. M., ANDERSONE, I., ANDERSONS, B. Heat treatment. In: Handbook of Wood Chemistry and Wood Composites. Boca Raton: CRC Press, pp.511-536, 2013.

[15] ROWELL, R. M., IBACH, R. E., MCSWEENY, J., et al., "Understanding decay resistance, dimensional stability and strength changes in heat-treated and acetylated wood", Wood Material Science and Engineering, v.1-2, p.14-22, 2009.

[16] WEIGL, M., MÜLLER, U., WIMMER, R., et al., "Ammonia vs. thermally modified timber-comparison of physical and mechanical properties", European Journal of Wood and Wood Products, v.70, p.233-239, 2012.

[17] AWOYEMI, L., WESTERMARK, U. "Effects of borate impregnation on the response of wood strength to heat treatment", Wood Science and Technology, v.39, pp.484-491, 2005.

[18] LAHTELA, V., TIMO KÄRKI, T. "Effects of impregnation and heat treatment on the physical and mechanical properties of Scots pine (Pinus sylvestris) wood", Wood Material Science \& Engineering, v.11, pp.217-227, 2014.

[19] SUN, B., WANG, X., LIU, J. "Changes in dimensional stability and mechanical properties of Eucalyptus pellita by melamine-urea-formaldehyde resin impregnation and heat treatment", European Journal of Wood and Wood Products, v.71, pp.557-562, 2013.

[20] PIZZI, A. "A new approach to nontoxic, wide-spectrum, ground-contact wood preservatives. Approach and reaction mechanisms", Holzforsch, v.47, pp.53-60, 1993.

[21] NGUYEN, T. T. H., LI, S., LI, J. "The combined effects of copper sulfate and rosin sizing agent treatment on some physical and mechanical properties of poplar wood", Construction and Building Materials, v.40, pp.33-39, 2013.

[22] TOMAK, E. D., VIITANEN, H., YILDIZ, U. C., et al., The combined effects of boron and oil heat treatment on the properties of beech and Scots pine wood. Part 2: Water absorption, compress, 2011.

[23] ASOCIACIÓN ESPAÑOLA DE NORMALIZACIÓN Y CERTIFICACIÓN (AENOR). Catálogo de Normas UNE EN 351-1- Durabilidad de la madera y de los productos derivados de la madera. Madera maci- 
za tratada con productos protectores. Parte 1: Clasificación de las penetraciones y retenciones de los productos protectores, 2010.

[24] AMERICAN SOCIETY FOR TESTING AND MATERIALS. ASTM D 2017. Standard method for accelerated laboratory test of natural decay resistance of woods. Philadelphia, 2005.

[25] MODES, K. S., LAZAROTTO, M., BELTRAME, R., et al., "Resistência natural das madeiras de sete espécies florestais 407 ao fungo Pycnoporus sanguineus causador da podridão-branca”, Cerne, v.18, pp.407411, 2012.

[26] RODRÍGUEZ, J. F. C., CHANES, J. W., PALACIOS, A. J., et al., "Influence of high pressure processing and alcaline treatment on sugarcane bagasse hydrolysis", Journal of Food, v.14, pp.613-620, 2015.

[27] CROITORU, C., PATACHIA, S., LUNGULEASA, A. "A mild method of wood impregnation with biopolymers and resins using 1-ethyl-3-methylimidazolium chloride as carrier", Chemical Engineering Research and Materials, 2015.

[28] LESAR, B., PAVLIC, M., PETRIC, M., et al., "Wax treatment of wood slows photodegradation", Polymer degradation and stability, v.96, pp.1271-1278, 2011.

[29] CAETANO, R. Breu (Agente de Pegajosidade): Ciência e Tecnologia da Borracha. 2014. Disponível em: <http://www.ctb.com.pt/?page_id=7163>. Acesso em 23 nov. 2014.

[30] WINDEISEN, E., STROBEL, C., WEGENER, G. "Chemical changes during the production of thermotreated beech wood”, Wood Science and Technology, v.41, pp.523-536, 2007.

[31] ZHANG, Y. M., YU, Y. L., YU, W. J. "Effect of thermal treatment on the physical and mechanical properties of Phyllostachys pubescen bamboo", European Journal of Wood and Wood Products, v.71, p.6167, 2013.

[32] WANG, X., YUHE, D., SIQUN, W., et al., "Evaluation of the effects of compression combined with heat treatment by nanoindentation (NI) of poplar cell walls", Holzforschung, v.68, pp.167-173, 2014.

[33] DINEFF, P., GOSPODINOVA, D., AVRAMOV, I. 2011. "Plasma aided flame retardation of wood, woodenproducts and cellulosic materials", Advances in Materials Sciences, v.11, p.28-42, 2011.

[34] YILDIZ, S., GEZER, E. D., YILDIZ, U. C. "Mecanical and chemical behavior of spruce wood modified by heart", Building and Environment, v.41, p.1762-1766, 2006.

[35] CALLISTER JR, W. D. Ciência e Engenharia de Materiais - Uma Introdução., 7.ed. Rio de Janeiro: LTC, 2008. 